\title{
Sistema de control de tickets para la empresa Kliente Strategik
}

fecha de recepción: 10-12-2020 • Fecha de aceptación: 26-01-2021 • Fecha de publicación: 10-02-2021

Danny Alexander Cárdenas Hidalgo ${ }^{1}$

Kliente Strategik, Ecuador

dach19962012@gmail.com

https://orcid.org/0000-0001-5658-8825

Víctor Adrián Guadalupe Ontaneda ${ }^{2}$

Metropolitan Touring, Ecuador vguadalupe@gmail.com

https://orcid.org/0000-0001-8341-840X

Pablo Mauricio Salazar Mora ${ }^{3}$ MEGA JB, Ecuador pablosalazar66@hotmail.com https://orcid.org/0000-0002-1573-3384

Bryan Alexis Vergara Castillo 4 Embajada de México, Ecuador bryanvcas@gmail.com https://orcid.org/0000-0002-3791-7256

\section{RESUMEN}

Actualmente la empresa Kliente Strategik maneja un sistema no automatizado para el control de tickets, lo cual ocasiona una pérdida de tiempo en el control de proceso y seguimiento de actividades. Este trabajo presenta el desarrollo de un sistema de control de tickets con una herramienta tecnológica diseñada para administrar tareas de soporte que actualmente son registradas manualmente. Los usuarios podrán monitorear la información de solicitudes y sus estados en tiempo real, dentro del 
sistema. La aplicación tiene una interfaz amigable, cumpliendo con estándares de UX y UI para una mejor experiencia y eficiencia en la gestión. Los resultados demuestran que esta aplicación permitió gestionar de forma adecuada las actividades operativas referentes al monitoreo de tickets.

PALABRAS CLAVE: gestión de tickets, seguimiento, interfaz, aplicación, automatización.

\section{ABSTRACT}

Currently the company Kliente Strategik handles a non-automated system for ticket control, which causes a loss of time in process control and monitoring of activities. This work presents the development of a ticket control system with a technological tool designed to manage support tasks that are currently recorded manually. Users will be able to monitor ticket information and its status in real time, within the system. The application has a friendly interface, complying with UX and UI standards for a better experience and efficiency in management. The results show that this application allowed to properly manage the operational activities related to ticket monitoring. 


\section{Introducción}

Las primeras aplicaciones móviles se crearon a partir de los años 2000 como una necesidad para hacer uso de las primeras aplicaciones de juegos, calendarios o agendas que ya estaban integrados en los dispositivos móviles (Martinez, 2001).

La evolución de estas avanza a pasos agigantados gracias a las innovaciones tecnológicas en cuanto a protocolos de aplicaciones inalámbricas (WAP, por sus siglas en inglés), esto viene acompañado de un desarrollo muy fuerte de los celulares. Apple lanzó el iPhone y junto a él llegaron muchas más propuestas de Smartphones, entre ellas Android. Por lo tanto, es allí que empieza el avance importante de las aplicaciones, ya sean de juegos, noticias, diseño, arte, fotografía, medicina, todo en tus manos gracias a la revolución de las aplicaciones móviles (Vanegas, 2012).

En la actualidad, el acceso a Internet y la tecnología de los dispositivos móviles permiten el desarrollo y consumo de aplicaciones. Existen gratuitas y de pago, así como didácticas y con metodologías como la gamificación, con la que vemos opciones mucho más interactivas.

En el contexto por la pandemia CODIV-19 y el salto tecnológico que muchos usuarios debieron realizar al verse obligados a no ir a una tienda física, muchas aplicaciones se han creado para satisfacer estas necesidades que por el distanciamiento nos vemos obligados a usar. Por citar ciertos ejemplos, se menciona a Silva et al. (2018) que desarrollan una app móvil para potenciar el turismo, por otro lado Reyes Rivera et al. (2020) crean una app de compras con servicio de entrega a domicilio en el contexto ecuatoriano, para la ciudad de quito.

En este trabajo se propone el desarrollo de una aplicación móvil para la empresa Kliente Strategik (Kliente Stratégik, n.d.), un aplicativo que permita realizar la administración y seguimiento de tickets de cada solicitud de soporte, agilizando la atención y monitoreo desde y hacia cualquier lugar geográficamente hablando, actualmente la empresa no cuenta con un proceso eficiente para el control de solicitudes de soporte, se realiza manualmente, desde que el cliente llama o se acerca a la empresa, verificar la disponibilidad, realizar el ingreso en una bitácora por cliente que se registra en la empresa. Esto acarrea una serie de fallos humanos como tiempos largos de atención, generando clientes insatisfechos con la atención, así como pérdida de información.

El objetivo de la solución planteada es que pueda adoptar una metodología más eficiente, ordenada y eficaz. Con esto se pretende brindar un servicio integral que satisfaga al cliente y modernizando la experiencia. En el proyecto se identificó el problema, la situación actual, justificación del tema, solución y alcance de este. Con lo cual se determinan los objetivos generales y específicos para luego obtener la especificación de los resultados esperados.

\section{Metodología}

El presente estudio busca automatizar procesos en la gestión de tickets que se vienen realizando de forma manual, por lo tanto, se utiliza el método deductivo (Castellanos, 2017) para llegar a al 
producto final, en base a las siguientes premisas:

- Los sistemas informáticos permiten automatizar procesos.

- Este trabajo propone el desarrollo de un sistema informático.

- El sistema propuesto automatizara el proceso de gestión de tickets

Para lo cual se desarrolla una aplicación para dispositivos móviles con sistema operativo Android (Android | The Platform Pushing What's Possible, n.d.) desde la versión 8.0 en adelante, la misma que interactuará con un Servicio Web REST API (Verborgh et al., 2014) con el formato de datos JSON (Pezoa et al., 2016), mismo que permitirá intercambiar datos con otras aplicaciones o servicios como con la base de datos, desde cualquier lugar del mundo gracias al Internet. La Figura 1 muestra el esquema de la aplicación.

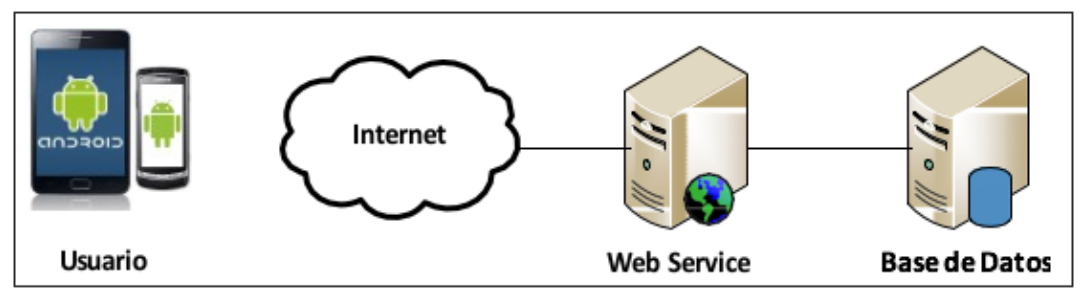

Figura 1. Esquema aplicación

Fuente: elaboración propia

Cabe aclarar que el uso de la aplicación se limitará solo para personal de la empresa, más concretamente para el personal de operaciones. Este les permitirá:

- Ingresar con su respectivo usuario y contraseña.

- Tener un menú amigable de tickets.

- Crear

- Modificar

- Almacenar

- Editar

- Eliminar

El servicio web implementado permitirá administrar de una forma ordenada los datos tanto en la actualización, edición y almacenamiento del detalle de cada ticket, usuarios e informes. Esta información estará disponible también en una interfaz de acceso Web donde los supervisores tendrán acceso y visibilidad de cada ticket y podrán darle seguimiento desde cualquier ubicación 
La Tabla 1 muestra el equipo de desarrollo de la aplicación propuesta utilizando la metodología de desarrollo SCRUM (Amaya Balaguera, 2015).

Tabla 1.

Equipo de trabajo

\begin{tabular}{|c|c|}
\hline Product Owner & Integrantes del grupo \\
\hline Equipo de Desarrollo & Integrantes del grupo \\
\hline Scrum Master & Danny Cárdenas \\
\hline Diseño & Adrián Guadalupe \\
\hline Reportes & Bryan Vergara \\
\hline Webservice & Pablo Salazar \\
\hline
\end{tabular}

Fuente: elaboración propia

\subsection{Desarrollo}

Se busca digitalizar y automatizar el proceso por el cual se gestionan los tickets de forma manual en una bitácora levantada en un documento de Excel. Al crear una interfaz que permita almacenar toda la información correspondiente a un ticket de manera directa a un sistema con flujos preestablecidos de trabajo y escalamiento.

Se utilizaron herramientas de última generación para el desarrollo, como:

- Xamarin (Xamarin I Open-Source Mobile App Platform for .NET, n.d.)

- Mysql (MySQL, n.d.)

El sistema creado requiere un enlace a una base de datos para presentar a los usuarios la información justa y necesaria para sus tareas dentro de la aplicación.

A continuación, se presenta el diseño preliminar, utilizando la herramienta de prototipado Balsamiq (Balsamiq. Rapid, Effective and Fun Wireframing Software I Balsamiq, n.d.). 


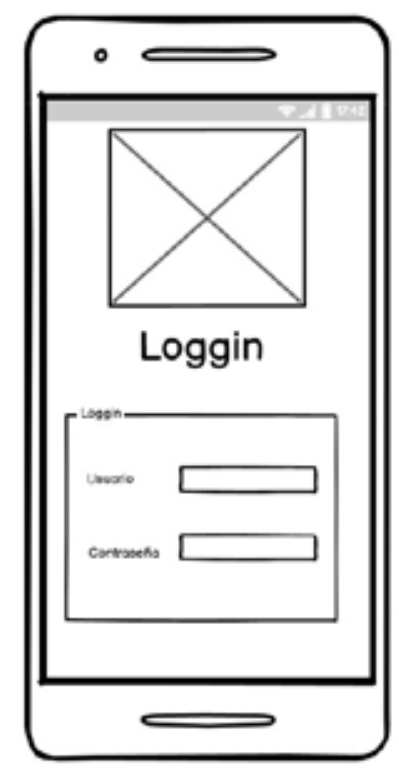

Figura 2. Login

Fuente: Balsamiq Wireframes

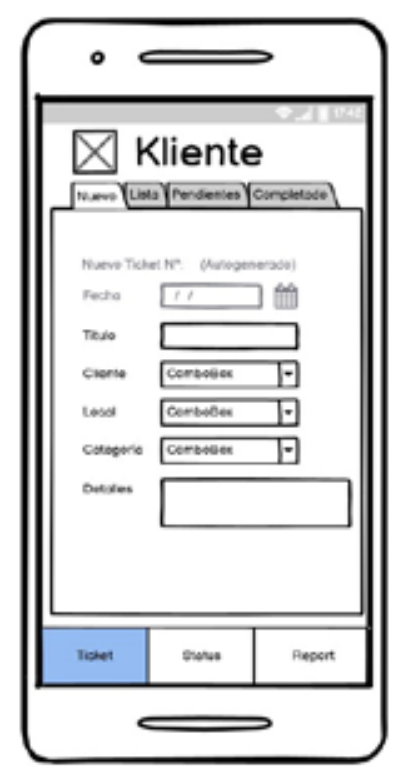

Figura 3. Ingreso Ticket Fuente: Balsamiq Wireframes

En la Figura 2 se puede visualizar un prototipo de la ventana de ingreso y en la Figura 3 se observa una ventana que permitirá registrar los nuevos tickets solicitados.

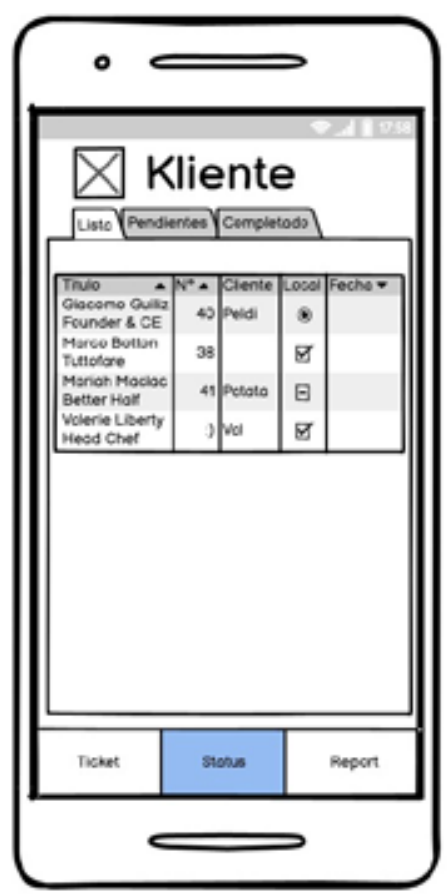

Figura 4. Lista de tickets

Fuente: Balsamiq Wireframes 


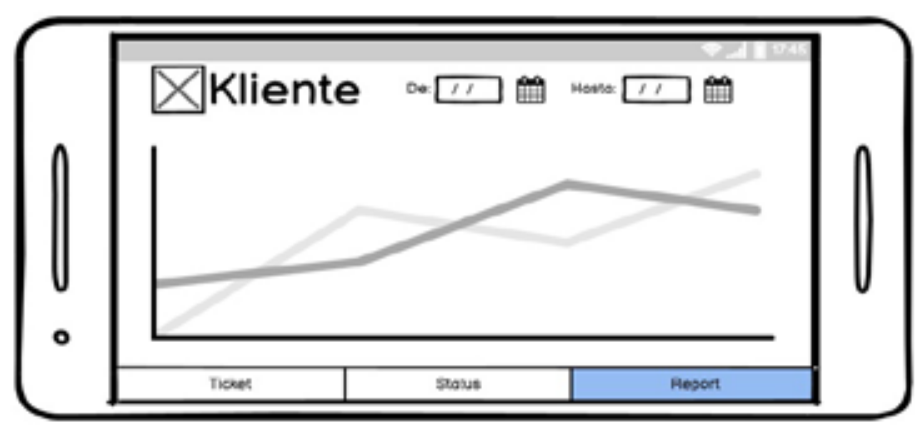

Figura 5. Chart

Fuente: Balsamiq Wireframes

En la Figura 4 se puede ver una lista con todas las tareas creadas y sus respectivos detalles y en la Figura 5 los gráficos estadísticos de los datos.

Las pruebas funcionales determinaron que se cumplen los requisitos del cliente y el sistema funciona de manera óptima. Se manejan eficientemente los hilos de procesamiento para no provocar pérdida de información y cuellos de botella al momento de actualizar los detalles de los tickets.

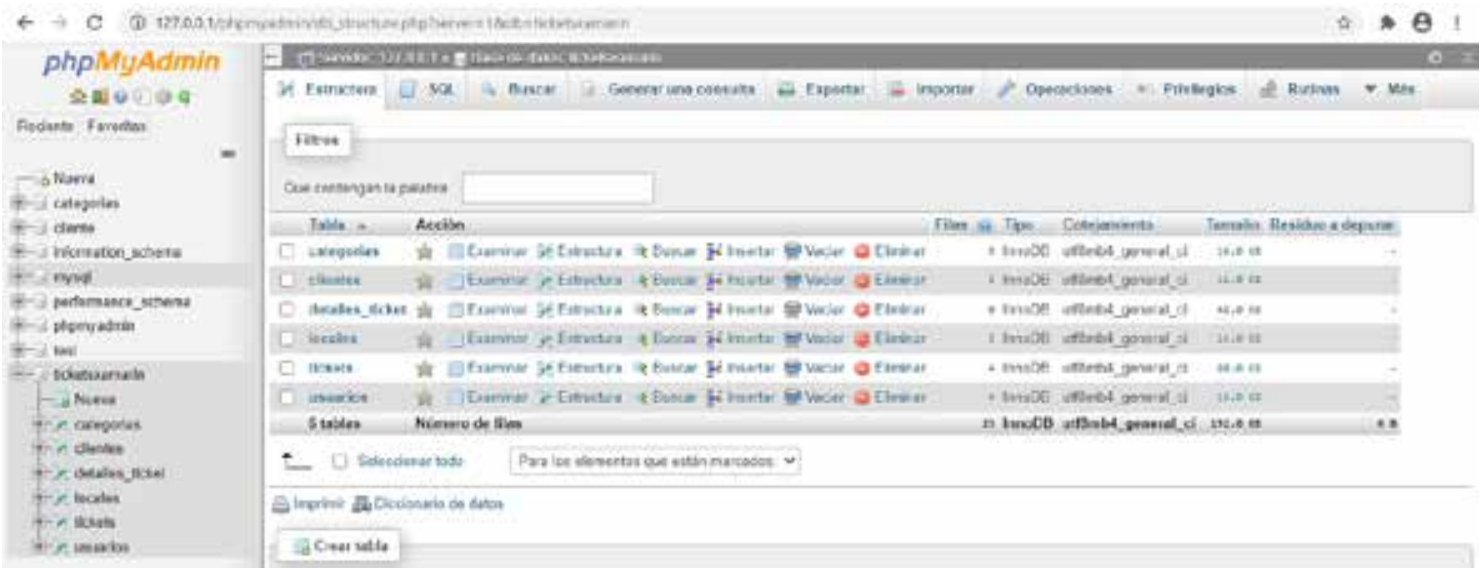

Figura 6. Base de datos

Fuente: $M y S Q L$

La Figura 6 muestra el modelo de base de datos utilizado en esta aplicación. 


\section{Resultados}

Una vez finalizada la fase de codificación y para la comprobación del correcto funcionamiento de la aplicación en cada uno de sus Menús, se realizaron las pruebas respectivas tomando en cuenta los aspectos siguientes:

- Para la primera prueba funcional se elaboró una pequeña síntesis sobre las "historias de usuarios" al momento de utilizar la aplicación, para lo cual se ha tomado en cuenta una Autenticación Correcta y una Autenticación Incorrecta de usuario al momento de ingresar.

- En segundo lugar, se probó la aplicación en un Smartphone que cumple los requisitos mínimos de la aplicación. Se realizó con empleados de la empresa Kliente Strategik y con datos reales para ver los tiempos de respuesta.

Esta es una pruebas funcional relacionada a las historias de usuario referentes a los Usuarios autenticados

Tabla 2.

Historial de usuario

\begin{tabular}{|l|ll|}
\hline \multicolumn{2}{|l|}{ Historia de usuario: Autenticación correcta de usuarios. } \\
\hline Descripción & $\checkmark \begin{array}{l}\text { El usuario una vez que ha ejecutado la aplicación previamente } \\
\text { instalada le aparecerá una la Ventana de "Inicio de Sesión", donde } \\
\text { ingresar su Usuario y la Contraseña, internamente se verificará } \\
\text { si estos datos son los correctos y posteriormente ingresará a la } \\
\text { aplicación. }\end{array}$ \\
\hline $\begin{array}{l}\text { Condiciones } \\
\text { de ejecución }\end{array}$ & $\checkmark$ & El usuario deberá estar registrado en el sistema. \\
\hline Entrada & $\checkmark$ & $\begin{array}{l}\text { El usuario ingresará su usuario y la contraseña y presionará el } \\
\text { botón Ingresar. }\end{array}$ \\
\hline $\begin{array}{l}\text { Resultado } \\
\text { esperado }\end{array}$ & $\checkmark$ & $\begin{array}{l}\text { Internamente se verificará si el usuario está registrado e ingresará } \\
\text { a realizar sus consultas. }\end{array}$ \\
\hline $\begin{array}{l}\text { El proceso de autenticación se considera finalizado. } \\
\text { correcto en la base de datos se registrarán datos de su ingreso y el } \\
\text { usuario podrá utilizar la aplicación que está cargada de información } \\
\text { de ese usuario especifico. }\end{array}$ \\
\hline de la prueba
\end{tabular}

Fuente: Elaboración propia 
Para la segunda prueba funcional, se realizó en un Smartphone (ver Figura 7) de las siguientes características:

- Samsung Smartphone

- Dual Core 1.2 GHz de $1 \mathrm{~GB}$ de RAM

- Sistema Operativo Android 6

Los usuarios de la aplicación pudieron constatar del funcionamiento de cada una de las funciones programadas, lo que permitió verificar el cumplimiento de los requisitos planteados al inicio de este proyecto.

Nos adentramos a un campo subvalorado en el diseño de aplicaciones como lo es la experiencia de usuarios con la aplicación (UX, por sus siglas en inglés) en la gráfica de la interfaz de usuario (UI, por sus siglas en inglés), que mejoran el manejo de la aplicación y sus funciones dentro de la interfaz. El resultado fue una aplicación con colores coherentes, fácil de usar, muy intuitiva y llamativa para el usuario que encontrará los controles y la navegación a unos pocos clics.

El resultado de estas pruebas lo veremos en las Figuras siguientes:

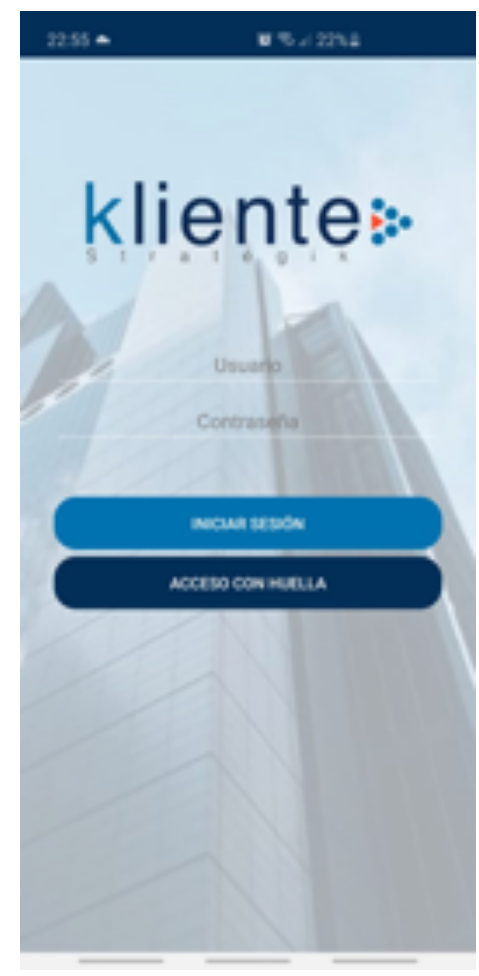

Figura 7. Ingreso de usuarios Fuente: aplicación Android

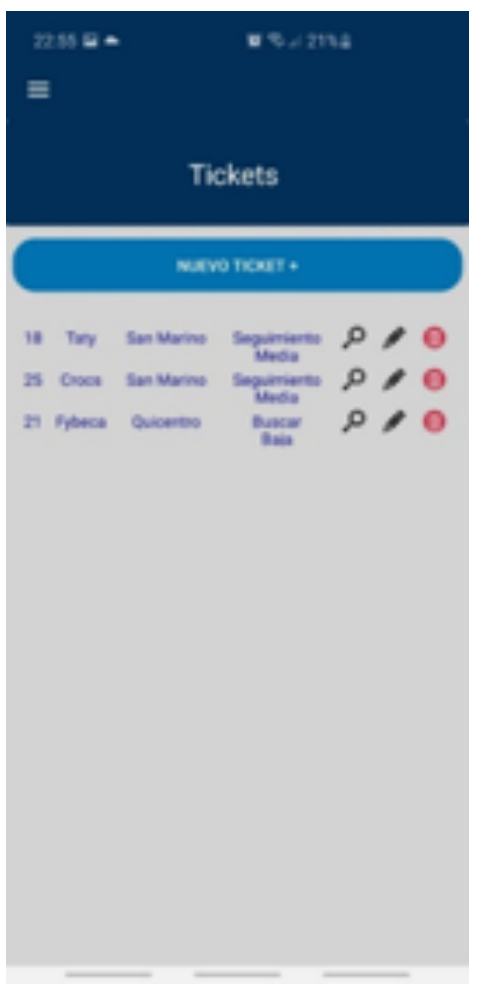

Figura 8. Ingreso ticket Fuente: aplicación Android 


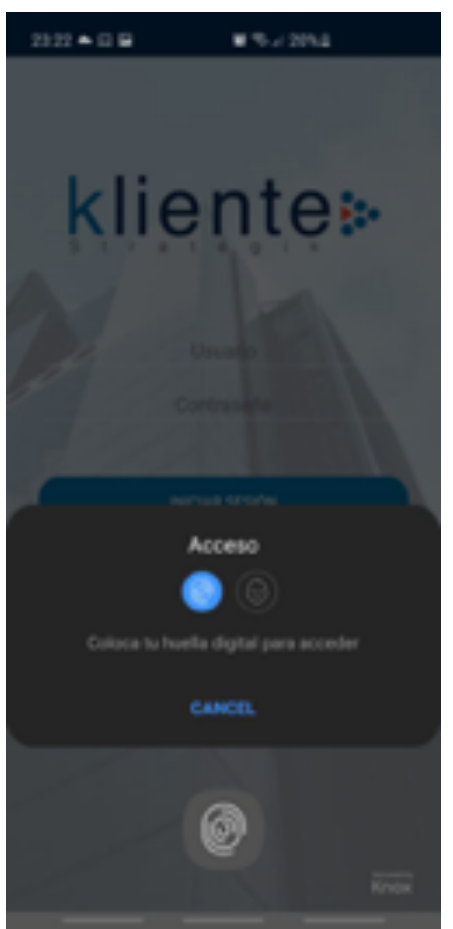

Figura 9. Ingreso con huella Fuente: aplicación Android

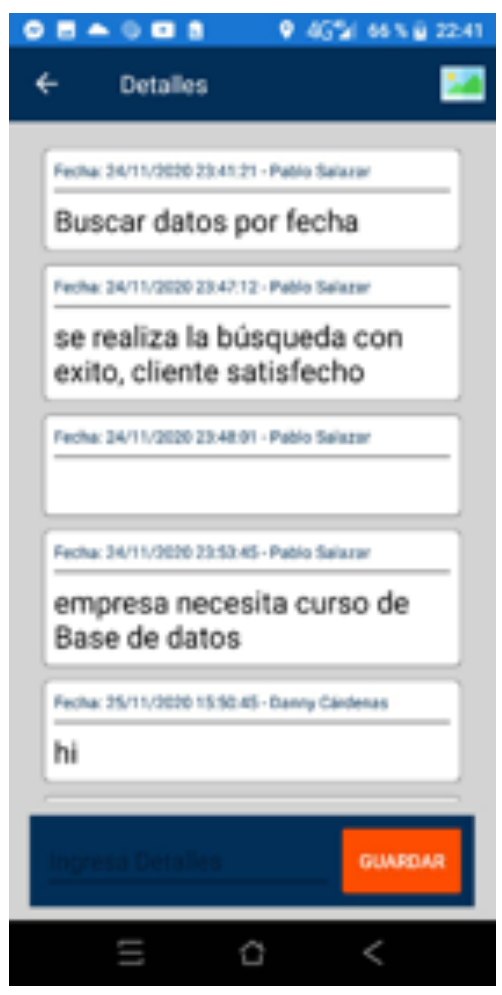

Figura 11. Detalle

Fuente: aplicación Android

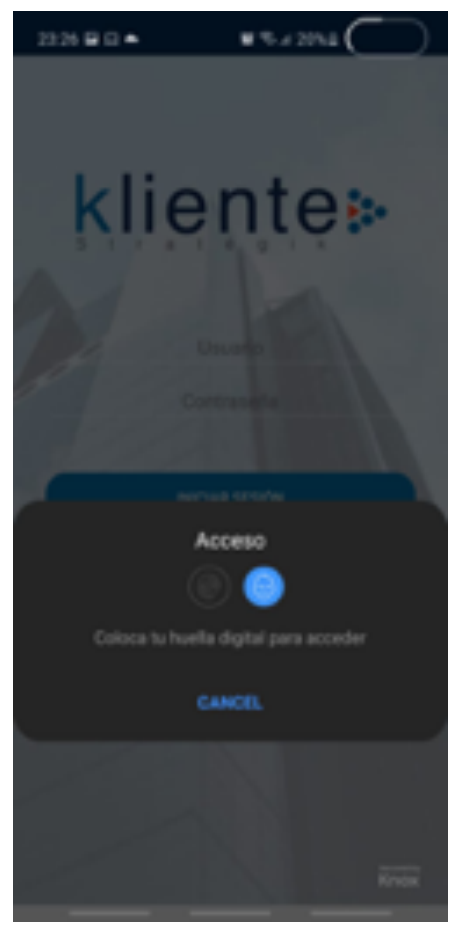

Figura 10. Ingreso con reconocimiento facial Fuente: aplicación Android

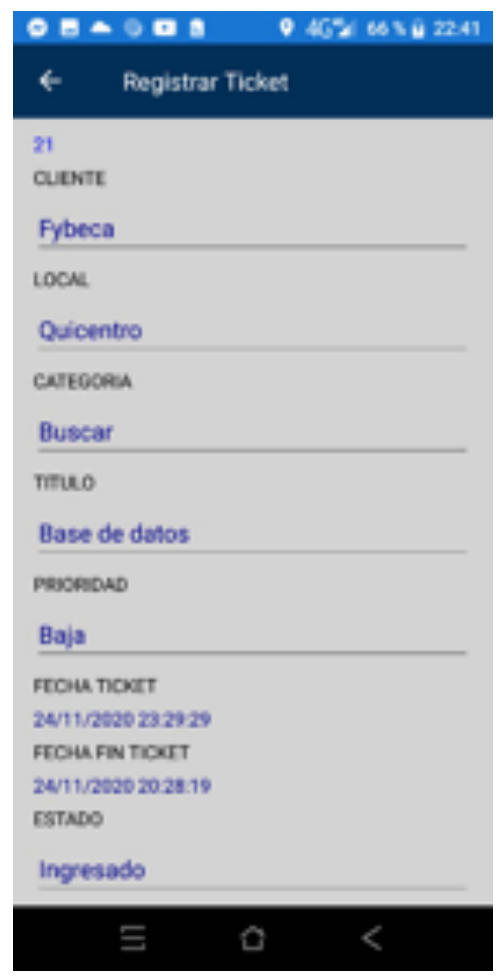

Figura 12. Registro ticket Fuente: aplicación Android 


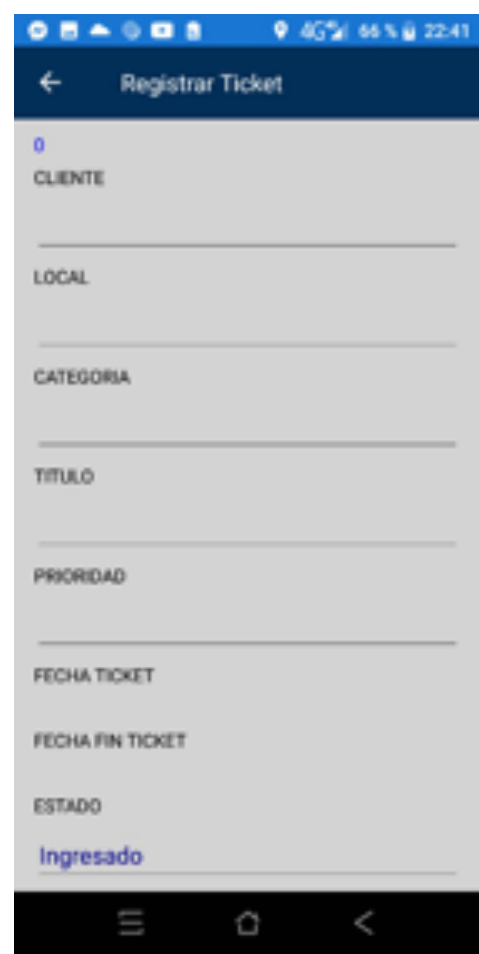

Figura 13. Registro ticket Fuente: aplicación Android

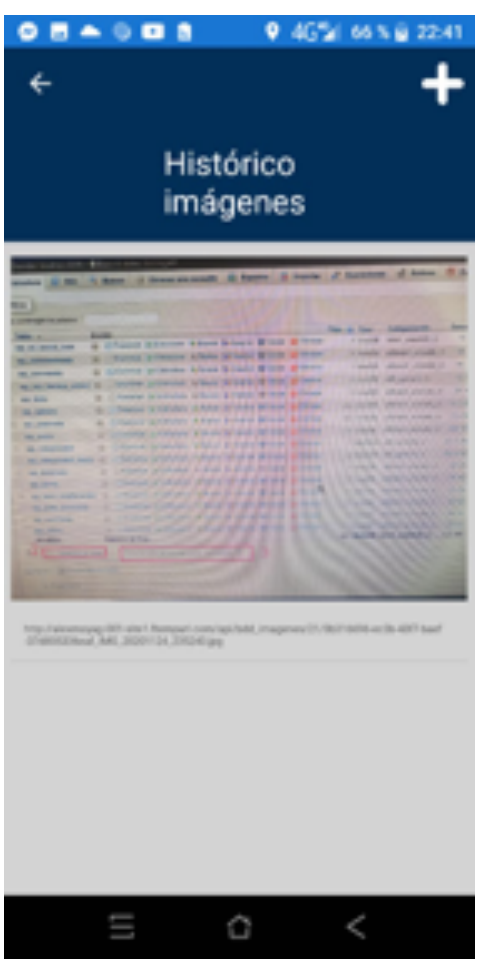

Figura 14. Histórico imágenes Fuente: aplicación Android

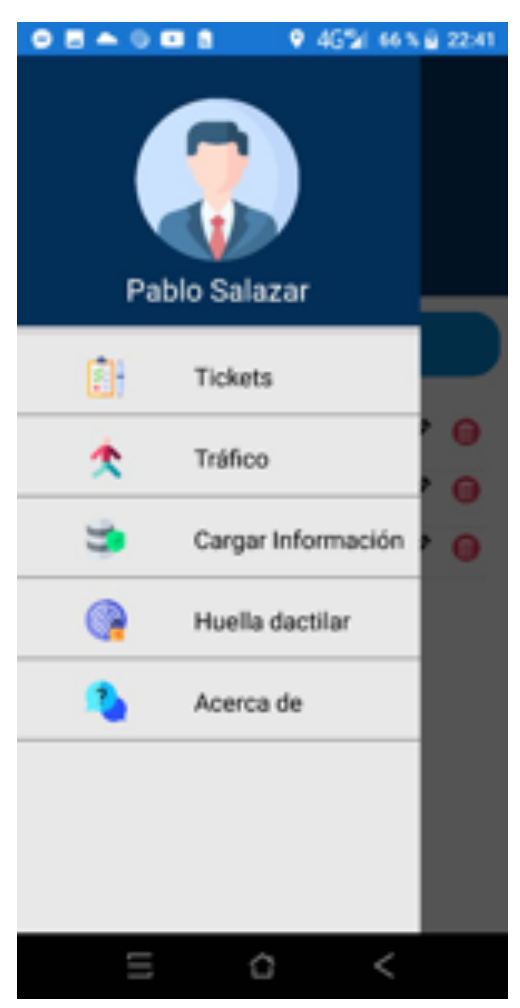

Figura 15. Menú

Fuente: aplicación Android 


\section{Conclusiones}

Al realizar una aplicación Android se aprovechó la gran cantidad de usuarios que disponen de Tablets y Smartphones con este sistema operativo donde se puede instalar y el usuario está familiarizado con su funcionamiento nativo.

Para el presenta artículo se utilizó Extreme Programming como metodología ágil completando cada una de sus etapas y permitiendo obtener varias versiones funcionales que fueron presentadas al usuario para su evaluación y corrección hasta obtener una versión final que cumplía con los requerimientos del cliente.

Como conclusiones, se obtuvo que la aplicación móvil redujo los tiempos de respuesta en los tickets y pudo acercar al cliente a un sistema eficiente y con alta disponibilidad para sus requerimientos. 


\section{Referencias}

Amaya Balaguera, Y. D. (2015). Metodologías ágiles en el desarrollo de aplicaciones para dispositivos móviles. Estado actual. Revista de Tecnología, 12(2). https://doi.org/10.18270/rt.v12i2.1291

Android / The platform pushing what's possible. (n.d.). Retrieved November 26, 2020, from https://www.android. $\mathrm{com} /$

Balsamiq. Rapid, Effective and Fun Wireframing Software / Balsamiq. (n.d.). Retrieved January 7, 2021, from https://balsamiq.com/

Castellanos, B. J. P. (2017). El uso de los métodos deductivo e inductivo para aumentar la eficiencia del procesamiento de adquisición de evidencias digitales. Cuadernos de contabilidad, 18(46).

Kliente Stratégik. (n.d.). Retrieved December 10, 2020, from https://kliente.one/

Martinez, E. (2001). La evolución de la telefonía móvil. Revista RES, 1, 1-6.

MySQL. (n.d.). Retrieved January 28, 2019, from https://www.mysql.com/

Pezoa, F., Reutter, J. L., Suarez, F., Ugarte, M., \& Vrgoč, D. (2016). Foundations of JSON schema. 25th International World Wide Web Conference, WWW 2016, 263-273. https://doi.org/10.1145/2872427.2883029

Reyes Rivera, A. J., Vallejos Cango, M. de L., \& Quintana García, D. A. (2020). Nueva realidad: compras en línea y a domicilio. REVISTA ODIGOS, 1(3), 47-60. https://doi.org/10.35290/ro.v1n3.2020.370

Silva, C. A., Toasa, R., Guevara, J., Martinez, H. D., \& Vargas, J. (2018). Mobile Application to Encourage Local Tourism with Context-Aware Computing. The 2018 International Conference on Information Technology \& Systems, ICITS'18, 796-803. https://doi.org/10.1007/978-3-319-73450-7_75

Vanegas, C. A. (2012). DESARROLLO DE APLICACIONES SOBRE ANDROID. Revista Vínculos, 9(2), 129145. https://doi.org/10.14483/2322939X.4275

Verborgh, R., Harth, A., Maleshkova, M., Stadtmüller, S., Steiner, T., Taheriyan, M., \& Van De Walle, R. (2014). Survey of semantic description of REST APIs. In REST: Advanced Research Topics and Practical Applications (Vol. 9781461492993, pp. 69-89). Springer New York. https://doi.org/10.1007/978-1-4614-9299-3_5

Xamarin I Open-source mobile app platform for .NET. (n.d.). Retrieved November 26, 2020, from https://dotnet. microsoft.com/apps/xamarin 